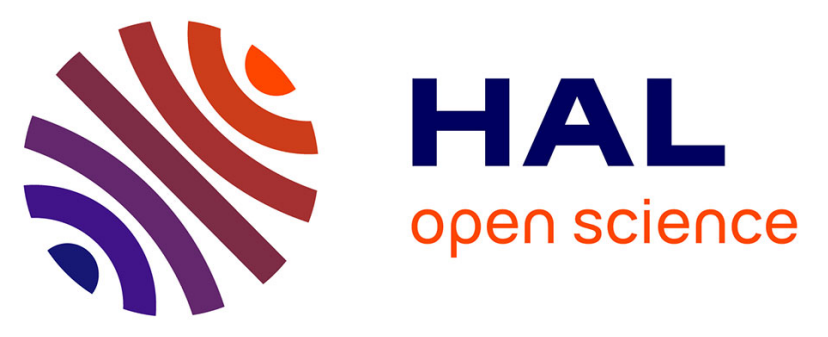

\title{
MR-ARFI-based method for the quantitative measurement of tissue elasticity: application for monitoring HIFU therapy
}

Jonathan Vappou, Pierre Bour, Fabrice Marquet, Valery Ozenne, Bruno

Quesson

\section{To cite this version:}

Jonathan Vappou, Pierre Bour, Fabrice Marquet, Valery Ozenne, Bruno Quesson. MR-ARFI-based method for the quantitative measurement of tissue elasticity: application for monitoring HIFU therapy. Physics in Medicine and Biology, IOP Publishing, 2018, 63 (9), pp.095018. 10.1088/1361$6560 /$ aabd0d . hal-03541175

\section{HAL Id: hal-03541175 \\ https://hal.archives-ouvertes.fr/hal-03541175}

Submitted on 24 Jan 2022

HAL is a multi-disciplinary open access archive for the deposit and dissemination of scientific research documents, whether they are published or not. The documents may come from teaching and research institutions in France or abroad, or from public or private research centers.
L'archive ouverte pluridisciplinaire HAL, est destinée au dépôt et à la diffusion de documents scientifiques de niveau recherche, publiés ou non, émanant des établissements d'enseignement et de recherche français ou étrangers, des laboratoires publics ou privés. 
Running Title: Quantitative MR-ARFI for HIFU therapy monitoring

\title{
MR-ARFI-based method for quantitative measurement of tissue elasticity: Application to monitoring of HIFU therapy
}

\author{
Jonathan Vappou ${ }^{1 *}$, Pierre Bour ${ }^{2,3,4,5}$, Fabrice Marquet ${ }^{2,3,4}$, Valery Ozenne 2,3,4 $^{2}$ and Bruno \\ Quesson $^{2,3,4}$ \\ ${ }^{1}$ ICube, UMR 7357 University of Strasbourg, CNRS, France \\ ${ }^{2}$ IHU Liryc, Electrophysiology and Heart Modeling Institute, Fondation Bordeaux Université, F- \\ 33600 Pessac- Bordeaux, France \\ ${ }^{3}$ Univ. Bordeaux, Centre de recherche Cardio-Thoracique de Bordeaux, U1045, F-33000, \\ Bordeaux, France \\ ${ }^{4}$ INSERM, Centre de recherche Cardio-Thoracique de Bordeaux, U1045, F-33000 Bordeaux, \\ France \\ ${ }^{5}$ Image Guided Therapy SA, Pessac, France
}

\section{(5468 words)}

* Jonathan Vappou, Ph.D ICube, University of Strasbourg, CNRS, France Mailing address: IRCAD/ICube, 1 place de l'Hôpital, 67091 Strasbourg , France Telephone: +33 388119132 Email: jvappou@unistra.fr 


\begin{abstract}
.
Monitoring thermal therapies thanks to medical imaging is essential in order to ensure that they are safe, efficient and reliable. In this paper, we propose a new approach, halfway between MR Acoustic Radiation Force Imaging (MR-ARFI) and MR Elastography (MRE), allowing for quantitative measurement of tissue's elastic modulus in a highly localized manner. It relies on the simulation of the MR-ARFI profile, which depends on tissue biomechanical properties, and on the identification of tissue elasticity through the fitting of the experimental displacement images measured using rapid MR-ARFI. Such method was specifically developed for the monitoring of MR-guided High Intensity Focused Ultrasound (MRgHIFU) therapy. Elasticity changes were followed during HIFU ablations $(\mathrm{N}=6)$ performed ex vivo in porcine muscle samples, and were compared to temperature changes measured by MR-Thermometry. Shear modulus was found to increase consistently and steadily a few seconds after the heating started, and such changes were found to be irreversible. The shear modulus was found to increase from $1.49 \pm 0.48 \mathrm{kPa}$ (before ablation) to $3.69 \pm 0.93 \mathrm{kPa}$ (after ablation and cooling). Thanks to its ability to perform quantitative elasticity measurements in a highly localized manner around the focal spot, this method was shown to be particularly interesting for the monitoring of HIFU ablations.
\end{abstract}




\section{Introduction}

Magnetic Resonance-guided High Intensity Focused Ultrasound (MRgHIFU) has emerged over the past decade as a highly valuable non invasive, non ionizing thermal therapy for a large variety of diseases, ranging from oncological (Gianfelice et al 2008, Schmitz et al 2008, Liberman et al 2009) to neurological diseases (Zaaroor et al 2017, Elias et al 2013). HIFU thermotherapy relies on the absorption of the acoustic energy by the tissue, leading to highly localized temperature elevation. Performing thermal ablations using HIFU therapy requires guidance by medical imaging for the targeting and the monitoring of thermal ablation in real time, as well as for assessing therapy efficiency post-operatively. MRI is particularly appreciated thanks to the constrast in soft tissues it offers intrinsically, to the possibility of imaging several planes freely with identical quality, and thanks to the possibility of monitoring the ablation in real time using MR Thermometry (Quesson et al 2000).

The monitoring of thermal ablations in real-time is essential in order to ensure that the target tissue is completely ablated while limiting unwanted damage to the surrounding sensitive structures and healthy tissue. MR thermometry based on the temperature dependence of the Proton Resonance Frequency (PRF) (Ishihara et al 1995, Rieke and Butts Pauly 2008) is currently the gold standard in non-fatty soft tissues, since it allows direct visualization of temperature distribution and computation of the accumulated thermal dose $(\mathrm{ThD})$ that serves as the therapy end point when a predefined threshold is reached (Sapareto and Dewey 1984). However, an uncertainty of a few degrees can lead to an overestimation of the thermal dose by an order of magnitude or more and therefore to an overestimation of the effective ablated zone. In the last 10 years, significant advances have been reported in preclinical research to accelerate and improve spatial coverage of PRF temperature mapping. MR thermometry with an uncertainty of $\sim 1{ }^{\circ} \mathrm{C}$ on abdominal organs (liver and kidney) has been reported by combining fast acquisition (echo planar imaging with parallel imaging) sequences with online motion compensation and correction of associated magnetic susceptibility artifacts (Roujol et al 2010, Mougenot et al 2009). Although it is a sophisticated and 
promising method, PRF-based MR thermometry cannot be performed in fatty tissues. This is particularly problematic for the treatment of breast tumors which are usually surrounded by fat or in the case of liver cancer for patients suffering from adipocytosis (Weidensteiner et al 2004). In these situations, precise monitoring from ThD imaging may become inaccurate. There is thus a clear lack of real-time monitoring methods that could offer additional types of information in complement to temperature/ThD estimate to account for structural changes during ablation. Tissue elasticity has been shown to be a promising biomarker for tissue changes related to heating. Both ultrasound and MR Elastography studies have demonstrated and quantified elasticity changes associated with thermal ablations (Mariani et al 2014, Corbin et al 2016, Chen et al 2013, Bing et al 2009, E. Konofagou et al 2012, Wu et al 2001, Larrat et al 2010), illustrating the promising value of elasticity imaging methods for the monitoring of thermal ablations. Magnetic Resonance Elastography (MRE) has been originally developed as a complementary method for the detection of abnormal tissue stiffening (Muthupillai et al 1996), based on the fact that most diseases are associated with substantial changes in biomechanical properties. MRE has shown to be highly valuable in a wide spectrum of clinical applications in oncology, hepatology, cardiovascular disease, or neurological diseases (Glaser et al 2012). Beyond its potential for diagnosis and disease staging, MRE has also been proposed for assessing thermal lesions. Liver tissue stiffening as a result of a laser ablation was demonstrated in vivo by Chen et al. (Chen et al 2013), in an experiment where MRE acquisitions were performed every 2 minutes during laser heating. A similar result was found both in vitro and in vivo by Corbin et al. (Corbin et al 2016) on liver tissue using real-time interventional MRE. Both skeletal muscle and turkey breast were shown to be stiffer after a HIFU ablation (Wu et al 2001, Larrat et al 2010), as opposed to brain tissue which was found to soften after HIFU treatment performed in vivo in rats (Larrat $e t$ al 2010). Monitoring HIFU ablations in real-time using MRE would be theoretically possible using realtime methods such as the one proposed by Corbin et al. (Corbin et al 2016) for laser ablations. However, such a solution would be particularly challenging and complex in terms of the experimental setup that requires both a mechanical exciter and the HIFU transducer to be positioned around the area of interest. 
Magnetic Resonance Acoustic Radiation Force Imaging (MR-ARFI) has been developed initially as a method for spotting the focal zone with high accuracy (McDannold and Maier 2008, Larrat et al 2008). Similarly to MRE, MR-ARFI relies on the encoding of tissue displacement on the MRI phase signal through the use of motion sensitizing gradients (MSG). Tissue displacement is the result of the acoustic radiation force acting at the focal zone. ARFI displacement-based methods are associated with significant limitations for HIFU therapy monitoring: Despite being correlated to tissue biomechanical properties, ARFI displacement cannot be considered as a reliable surrogate for its elasticity. It depends not only on local elasticity, but also on the mechanical properties of the surrounding tissue, on tissue heterogeneity, and on mechanical boundary conditions (Vappou et al 2015). MR-ARFI methods have been extended to the measurement of mechanical properties: either displacement can be used (Liu et al 2015, Dadakova et al 2017, Bour et al 2017) or shear wave velocity, which is related to tissue elasticity (Liu et al 2015, Souchon et al 2008). Shear wave velocity-based methods involve a spatial averaging effect: a minimal distance (ex: $\sim 10-20 \mathrm{~mm}$ (Liu et al 2015)) is needed in order to estimate properly the shear wave velocity. Therefore, these methods are very limited for measuring tissue elasticity specifically at the focal region, which is precisely where tissue structural (and biomechanical) changes are expected to be the most important. A 2015 study by Payne et al. (Payne et al 2015) aimed at simulating MR-ARFI displacement profiles using the Green's formalism for a point-source impulse. Computed displacements were found to be in good agreement with experimental ones on phantoms with varying stiffness. However, there was no direct estimation of tissue elasticity and the proposed mechanical information was not used for the monitoring of HIFU therapy. In this study, we propose a new approach for quantitative estimation of tissue elasticity at the focal zone, using the identification of the MR-ARFI profile. Theoretical background of the proposed method is presented and validation of the identification process is performed on numerical phantoms. Finally, experimental results obtained on ex vivo muscle undergoing HIFU ablations are presented. 


\section{Methods}

\section{Theoretical background: Relationship between the MR-ARFI profile and}

\section{tissue mechanical properties}

The method used for estimating biomechanical properties is based on a homemade 2D MR-ARFI push simulator that computes the MRI phase profile as a function of experimental parameters. As it has already been described in MR Elastography, a phase shift is observed when the tissue undergoes a displacement denoted as $\vec{d}$ simultaneously to a motion sensitizing magnetic field gradient (MSG) $\vec{G}$ of the MRI sequence. In $2 \mathrm{D}$, this can be written in polar coordinates:

$$
\Phi(r, \theta)=\gamma \int_{0}^{T_{M S G}} \vec{G}(t) \cdot \vec{d}(r, \theta, t) d t
$$

Where $\mathrm{T}_{\mathrm{MSG}}$ is the duration of the application of the motion-sensitizing gradient, and $\gamma$ the gyromagnetic ratio of hydrogen. Displacement is assumed to occur only in the direction perpendicular to the image slice. In practice, this is achieved by choosing a $2 \mathrm{D}$ slice orthogonal to the direction of the ARFI push with a MSG applied in the slice direction. In the model, the ARFI push is assumed to be punctual and localized at $\mathrm{r}=0$. This is obviously an important assumption that will be discussed later in this manuscript, since the actual displacement field is related to the dimensions and shape of the focal spot. In order to take tissue viscoelasticity into account, the displacement resulting from the ARFI push is supposed to follow a first order exponential law:

$$
\begin{cases}d(r=0, & t)=A\left(1-e^{-\frac{t}{\tau}}\right) \text { for } t \leq T_{p u s h} \\ d(r=0, & t)=A\left(e^{-\frac{t-T_{p u s h}}{\tau}}\right) \text { for } t \geq T_{p u s h}\end{cases}
$$


Where $T_{\text {push }}$ is the duration of the ARFI push, $\tau$ a time constant related to the material's viscoelasticity, and $\mathrm{A}$ the maximum displacement. In these equations, the push is assumed to start at $\mathrm{t}=0$.

This displacement results in the propagation of a shear wave propagating along the $r$ direction for each $\theta$ direction. In other words, the wavefront arrives at a specific position $r$ at a time $t=\frac{r}{c_{s}}$, where $\mathrm{c}_{\mathrm{s}}$ is the celerity of the shear wave. In a purely linearly elastic medium, the shear wave velocity is directly related to tissue's shear modulus $G$ and tissue density $\rho$ (supposed to be equal to $1000 \mathrm{~kg} \cdot \mathrm{m}^{-3}$ in soft tissues) by:

$$
c_{s}=\sqrt{\frac{G}{\rho}}
$$

Therefore, it is possible to compute the displacement field $d(\mathrm{r}, \theta, \mathrm{t})$ using both the relationships given by eqs. (2) and (3) and the knowledge of tissue elasticity given by its shear wave velocity $c_{s}$ or its shear modulus $G$. In this study, the tissue is supposed to be locally isotropic, i.e., the shear wave velocity, and therefore the displacement field do not depend on $\theta$. The proposed simulator aims at computing the MRI phase profile $\phi$ and uses as input the tissue elasticity (given by $c_{s}$ or $(G, \rho)$ ), the duration of the ARFI push $\mathrm{T}_{\text {push }}$, MSG duration $T_{M S G}$ and shape, as well as the size of the spatial window in which the phase profile has to be calculated. Tissue relaxation constant $\tau$ is fixed in this study and assumed to be equal to $4 \mathrm{~ms}$. As it will be discussed later in this manuscript, such an assumption is not expected to have a major impact on the elasticity estimate, since the ARFI profiles that are used for the identification are normalized in amplitude. Although the question of viscoelasticity is highly important from a biomechanical point-of-view, it is beyond the scope of this first study, which is focused on the identification of tissue elasticity only. The maximum push displacement was normalized $(A=1)$. Figure 1 illustrates an example of the phase profile for two different values of $c_{s}\left(c_{s}=1.5 \mathrm{~m} / \mathrm{s}\right.$ and $\left.c_{s}=2 \mathrm{~m} / \mathrm{s}\right)$, using bipolar rectangular MSG applied during 10 
$\mathrm{ms}$, and an ARFI push duration equal to $5 \mathrm{~ms}$. The influence of tissue elasticity can be clearly seen in the phase profile: qualitatively speaking, the size of the ARFI spot increases with tissue stiffness.
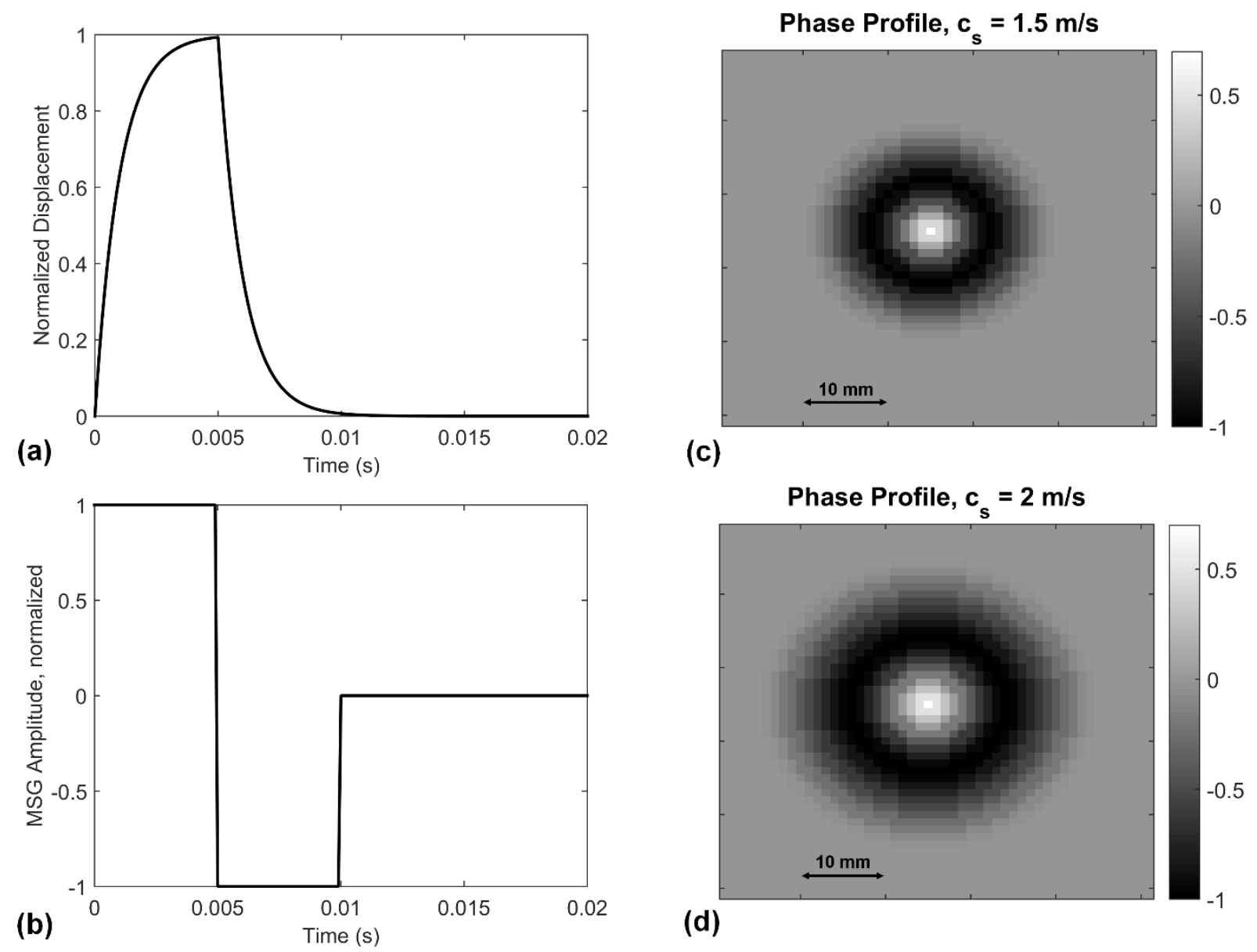

(c)

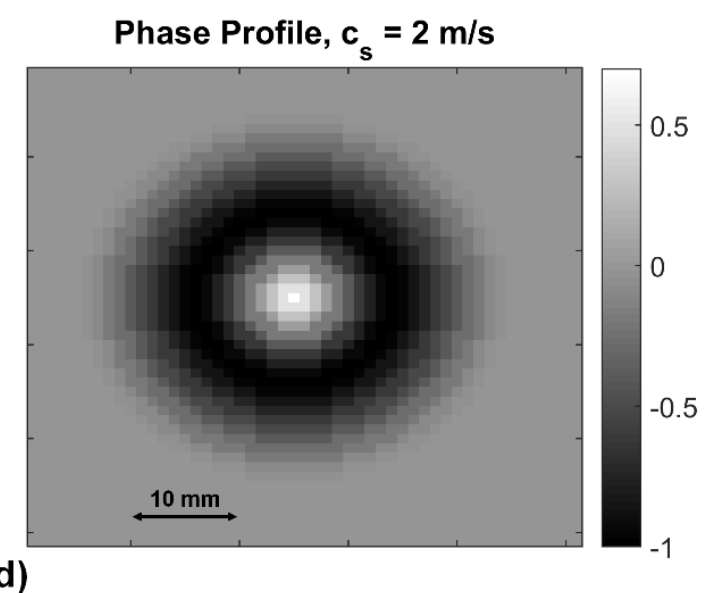

Figure 1: (a) Normalized displacement profile used for this example, with an ARFI push duration of 5 ms; (b) MSG profile used (bipolar rectangular); (c) Normalized phase profile obtained with $\mathrm{c}_{\mathrm{s}}=1.5 \mathrm{~m} / \mathrm{s}$; (d) Normalized phase profile obtained with $\mathrm{c}_{\mathrm{s}}=2 \mathrm{~m} / \mathrm{s}$

\section{Fitting process for elasticity estimation}

The fitting process is performed on normalized ARFI profiles centered on the focus, within a window of $51 \mathrm{~mm} * 51 \mathrm{~mm}$. Experimental ARFI profiles are interpolated by a factor of 2 in order to fit the resolution of the simulated ARFI profiles. The mean quadratic difference between experimental and simulated profiles 
is used as a cost function for different values of $c_{s}$. This cost function is minimized using a bounded optimization algorithm (fminbnd, Matlab, Mathworks), using an initial value of $1 \mathrm{~m} / \mathrm{s}$. The shear modulus is calculated subsequently using equation (4).

In order to evaluate the identification process, a numerical study was carried out on numerical phantoms with two different levels of added Gaussian noise ( $S N R=2, \mathrm{SNR}=5$ ). For each SNR level, tissue stiffness was varied between $\mathrm{cs}=1 \mathrm{~m} / \mathrm{s}$ and $\mathrm{cs}=2.4 \mathrm{~m} / \mathrm{s}$, which corresponds to a realistic range for shear wave velocity values in soft tissues. All other parameters were set identically for all simulations: ARFI push duration was set to $5 \mathrm{~ms}$, bipolar rectangular MSG were used (total duration $=10 \mathrm{~ms}$ ), pixel resolution of $1.5 \mathrm{~mm}$, and relaxation constant $\tau=4 \mathrm{~ms}$. For each case, 20 numerical phantoms were generated and the shear wave velocity was identified.

\section{Experimental protocol}

Ex vivo experiments were performed on a $1.5 \mathrm{~T}$ preclinical scanner (Siemens Aera, Erlangen, Germany) combined with an MR-compatible HIFU platform (Image-guided Therapy SA, Pessac, France). This platform consisted of a 256-elements phase array transducer (focal length $14 \mathrm{~cm}$, aperture $13 \mathrm{~cm}, 1 \mathrm{MHz}$ operating frequency). HIFU sonication was planned and executed from Matlab, using a custom library (IGTfus ,Image-guided Therapy SA, Pessac, France) which controls pulses durations, amplitudes and phases for each element. Two types of experiments were performed:

- The first experimental protocol aimed at validating the proposed MR-ARFI simulator. This was achieved by varying MR-ARFI parameters such as the delay between the MSG and the ARFI push (see $\mathrm{T}_{\text {Delay }}$ in Figure 2) and by comparing experimental MR-ARFI profiles to numerical ones. 
- The second experimental protocol aimed at monitoring HIFU ablations by following the elasticity estimated by the proposed method. Elasticity values were compared to temperature measured by MR-thermometry.

All experiments were performed on fresh ex vivo pig muscle samples. A Plexiglas tank was positioned on the bed of the HIFU transducer and filled with water at a temperature of $25^{\circ} \mathrm{C}$ (see Figure $2 \mathrm{~b}$ ). The sample was maintained in a plastic box. Mylar membranes (50 $\mu \mathrm{m}$ thick, smaller than $1 / 30$ of the ultrasonic wavelength) were glued to the base of the plastic box and water tank to ensure ultrasound coupling between the different interfaces. Optimal coil coverage was realized using two 32-element MR coils positioned on each side of the Plexiglas tank and an additional $19 \mathrm{~cm}$ loop coil positioned close to the sample.

MR data acquisition was performed using a gradient echo single-shot echo planar imaging (EPI) sequence (Bour et al 2017) where a bipolar MSG was added before the EPI echo train (Figure 2a). The acquisition slice was positioned in coronal orientation and MSG direction was set parallel to the acoustic propagation axis to encode longitudinal tissue displacement. In the sequence user interface, duration ( $\mathrm{T}_{\mathrm{MSG}}$ ) and amplitude $(G$, maximal value of $24 \mathrm{mT} / \mathrm{m})$ of MSG were adjustable parameters. In this protocol, the total duration and the amplitude of the $\mathrm{MSG}$ cycle were set to $\mathrm{T}_{\mathrm{MSG}}=10 \mathrm{~ms}$ and $\mathrm{G}=24 \mathrm{mT} / \mathrm{m}$, respectively. This sequence allows simultaneous MR Thermometry and MR-ARFI, similarly to what has already been reported in MRE and MR-ARFI (Le et al 2006, Auboiroux et al 2012).

Both experiments followed these steps:

1. Localization of muscle sample regarding to the HIFU transducer was performed using a 2D multislice balanced-SSFP sequence: FOV $=250 \times 250 \mathrm{~mm}^{2}, \mathrm{TR} / \mathrm{TE} / \mathrm{FA}=493 \mathrm{~ms} / 1.36 \mathrm{~ms} / 80^{\circ}, 1 \times 1 \times 3$ $\mathrm{mm}^{3}$ voxel size, with a bandwidth of $1149 \mathrm{~Hz}$ per pixel. 40 slices of each orientation centered on 
the transducer were acquired. From this stack of images, a target was selected and adjustments of the position of the monitoring slice were performed.

2. One coronal slice with fat saturation was acquired with the proposed sequence. Sequence parameters were: $\mathrm{FOV}=189 \times 189 \mathrm{~mm}^{2}, \mathrm{TR} / \mathrm{TE} / \mathrm{FA}=200 \mathrm{~ms} / 28 \mathrm{~ms} / 35^{\circ}$, voxel size $=1.5 \times 1.5 \times 5$ $\mathrm{mm}^{3}$, with a bandwidth of $1395 \mathrm{~Hz}$ per pixel, GRAPPA acceleration (factor = 2), partial Fourier $6 / 8, \mathrm{G}=24 \mathrm{mT} / \mathrm{m}, \mathrm{T}_{\mathrm{MSG}}=5 \mathrm{~ms}$ for the MSG. Each measurement was averaged five times to increase the precision of phase measurement, resulting in an actual refresh rate of $1 \mathrm{~Hz}$.

MR raw data were reconstructed using the open source framework Gadgetron (Hansen and Sørensen 2013). The reconstruction process encompasses EPI ghost correction using three acquisition of the central k-space line, coil compression and GRAPPA parallel imaging reconstruction. In both experiments, magnitude and phase images were transferred by TCP/IP to a visualization console (Thermoguide ${ }^{\mathrm{TM}}$, Image-guided Therapy SA, Pessac, France) for online computation and display of temperature and displacement maps, using a software module developed in-house (Bour et al 2017). The same console controlled the hardware of the HIFU device for selection of sonication parameters.

In the post-treatment stage for tissue elasticity identification, experimental displacement profiles were computed as follows: Phase difference images $\left(\varphi^{+}-\varphi^{-}\right)$were unwrapped spatially in $2 \mathrm{D}$ using the branch cut method proposed by Goldstein et al. (Goldstein et al 1988), and were normalized with respect to the maximum absolute displacement. The identification process was performed within a limited region around the focal spot $(5.1 \mathrm{~cm} * 5.1 \mathrm{~cm})$, on unwrapped, phase difference data. These normalized experimental displacement data were fitted by our MR-ARFI push simulator in order to identify tissue elasticity around the focal zone, as already explained in the numerical phantom study. 


\section{Experimental protocol \#1: Comparison study}

In this first experiment, experimental ARFI profiles were compared to simulated profiles at different values of the delay $\mathrm{T}_{\text {Delay }}$ between the MSG and the ARFI push. The HIFU shot $\left(\mathrm{S}_{\mathrm{ARFI}}\right)$ was synchronized with the MSG with an adjustable delay $\mathrm{T}_{\text {Delay }}$ set from the beginning of the MSG. The amplitude of the shot $\left(\mathrm{S}_{\text {THERMO }}\right)$ was turned off to avoid heating (see Figure 2a). The duration and acoustic power of $\left(\mathrm{S}_{\mathrm{ARFI}}\right)$ were set to $\mathrm{T}_{\text {Push }}$ $=2 \mathrm{~ms}$ and $447 \mathrm{~W}$, respectively. The delay $\mathrm{T}_{\text {Delay }}$ was varied between 0 and $9.5 \mathrm{~ms}$ by steps of $500 \mu \mathrm{s}$, for positive and negative MSG polarity. In other words, the MR-ARFI profile was measured for 20 equallyspaced phase shifts between the MSG and the ARFI push, resulting in 40 image frames with positive and negative displacement encoding. Temperature was monitored to control energy deposition during the procedure. The resulting MR-ARFI profiles were compared to those obtained by the proposed MR-ARFI simulator where $\mathrm{T}_{\text {Delay }}$ was varied as in the experimental protocol. Input parameters for the MR-ARFI simulator were: $\mathrm{MSG}$ duration $\mathrm{T}_{\mathrm{MSG}}=10 \mathrm{~ms}$, MSG shape $=$ square, duration of $\mathrm{ARFI}$ push $\mathrm{T}_{\text {push }}=2 \mathrm{~ms}$, size of window for simulation $=60 \times 60 \mathrm{~mm}^{2}$, viscoelasticity relaxation time $\tau=4 \mathrm{~ms}$, and shear wave velocity $\mathrm{c}_{\mathrm{s}}=1.1 \mathrm{~m} / \mathrm{s}$ corresponding to a shear modulus $\mathrm{G}=1210 \mathrm{~Pa}$. This elasticity value was set according to the result of the identification performed on one independent shot with $\mathrm{T}_{\text {Delay }}=0 \mathrm{~ms}$. 
a

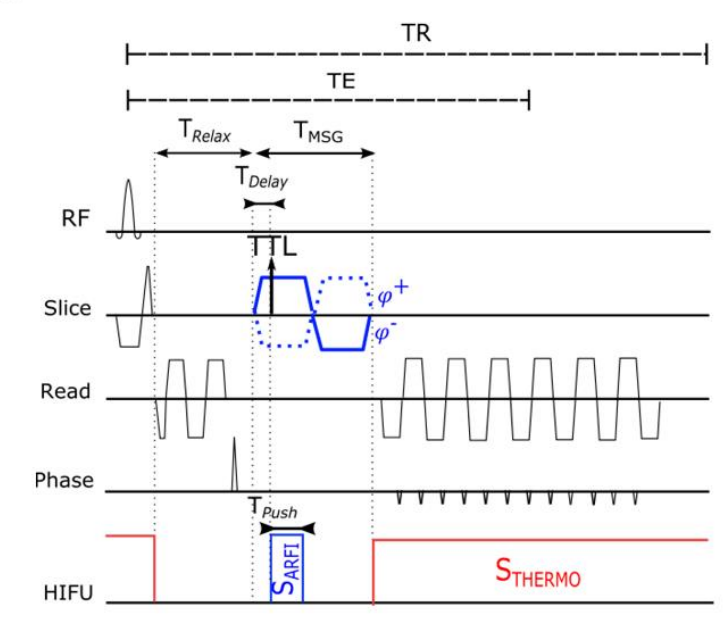

b

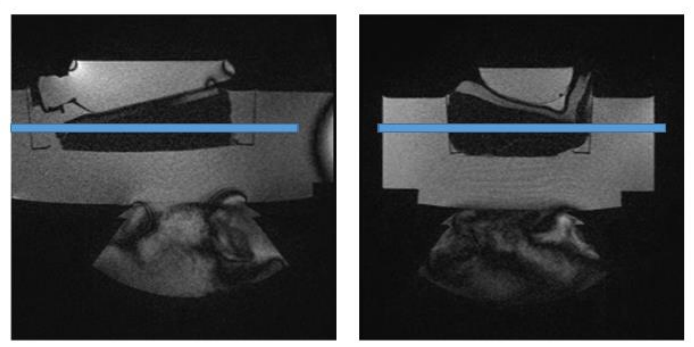

C

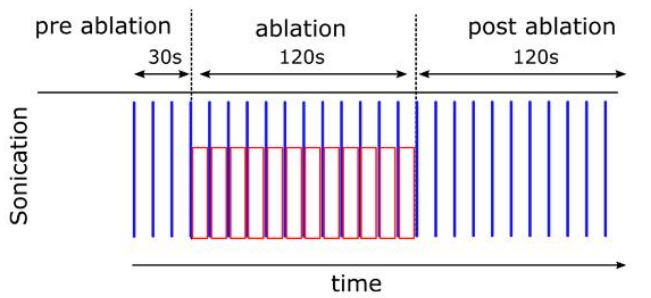

Figure 2: a) Chronogram of the single-shot echo planar imaging MR-ARFI sequence integrating MSG (in blue) with alternating polarities $\left(\varphi^{+}\right.$and $\left.\varphi^{-}\right)$. $\left(\mathrm{S}_{\mathrm{ARFI}}\right)$ represents the HIFU sonication of duration $\mathrm{T}_{\text {Push }}$ which produces the ARFI push. $\mathrm{T}_{\text {Delay }}$ and $\mathrm{T}_{\mathrm{MSG}}$ represent an adjustable synchronization delay and MSG total duration, respectively. TTL represents the synchronization pulse produced by the scanner.

b) 2D balanced-SSFP images acquired in sagittal (top) and transverse (bottom) of the experimental setup. Blue lines represents the acquisition slice centered on the HIFU focal location.

c) Typical sonication chronogram combining a pre-ablation, an ablation and a post-ablation phase. Blue box represent successive $\mathrm{S}_{\mathrm{ARFI}}$ shots all along the procedure and additional red box representing $\mathrm{S}_{\mathrm{THERMO}}$ shots during the ablation phase.

\section{Experimental protocol \#2: Monitoring of HIFU ablations}

In these experiments $(\mathrm{N}=6)$ performed on separate muscle samples, a second HIFU shot $\left(\mathrm{S}_{\mathrm{THERMO}}\right)$ was applied during the rest of the sequence, except during the second lobe of the MSG (Fig. 2(a)). An additional adjustable delay $\mathrm{T}_{\text {Relax }}=20 \mathrm{~ms}$ was set before the first lobe of the MSG in order to allow tissue mechanical relaxation after $\mathrm{S}_{\text {THERMO }}$ and thus avoiding residual displacement encoding. 
The sonication was divided in three phases (pre-ablation, ablation, and post-ablation) without interruption of the MR sequence (Fig. 2(c)):

1. Pre-ablation: $S_{\text {ARFI }}=447 \mathrm{~W}$ and $S_{\text {THERMO }}=0 \mathrm{~W}$ acoustic power, during $30 \mathrm{~s}$. This phase was used to characterize the tissue elasticity properties (young modulus) before ablation with a sonication duty cycle of $1 \%$ resulting in a limited energy deposition.

2. Ablation: $\mathrm{S}_{\mathrm{ARFI}}=447 \mathrm{~W}$ and $\mathrm{S}_{\mathrm{THERMO}}=155 \mathrm{~W}$ acoustic power, during $120 \mathrm{~s}$. During this phase, acoustic energy deposition was increased by the use of $\mathrm{S}_{\text {THERMO }}$ to reach a sonication duty cycle of $86 \%$ in order to induce a thermal lesion.

3. Post-ablation: $\mathrm{S}_{\mathrm{ARFI}}=447 \mathrm{~W}$ and $\mathrm{S}_{\mathrm{THERMO}}=0 \mathrm{~W}$ acoustic power, during the rest of the acquisition time. This phase was performed to observe the cooling period and evolution of elasticity after ablation.

Temporal variations of tissue elasticity were compared to those of the temperature measured by MR Thermometry.

\section{Results}

\section{Numerical phantoms}

Figure 3 illustrates the results for the identification process carried out at two different SNR levels, for shear wave velocity values ranging from $c_{s}=1 \mathrm{~m} / \mathrm{s}$ and $c_{s}=2.4 \mathrm{~m} / \mathrm{s}$. For each value of shear wave velocity, 20 numerical phantoms were generated and the shear wave velocity was estimated. Dots and errorbars denote the average and standard deviation across these 20 cases, respectively. 

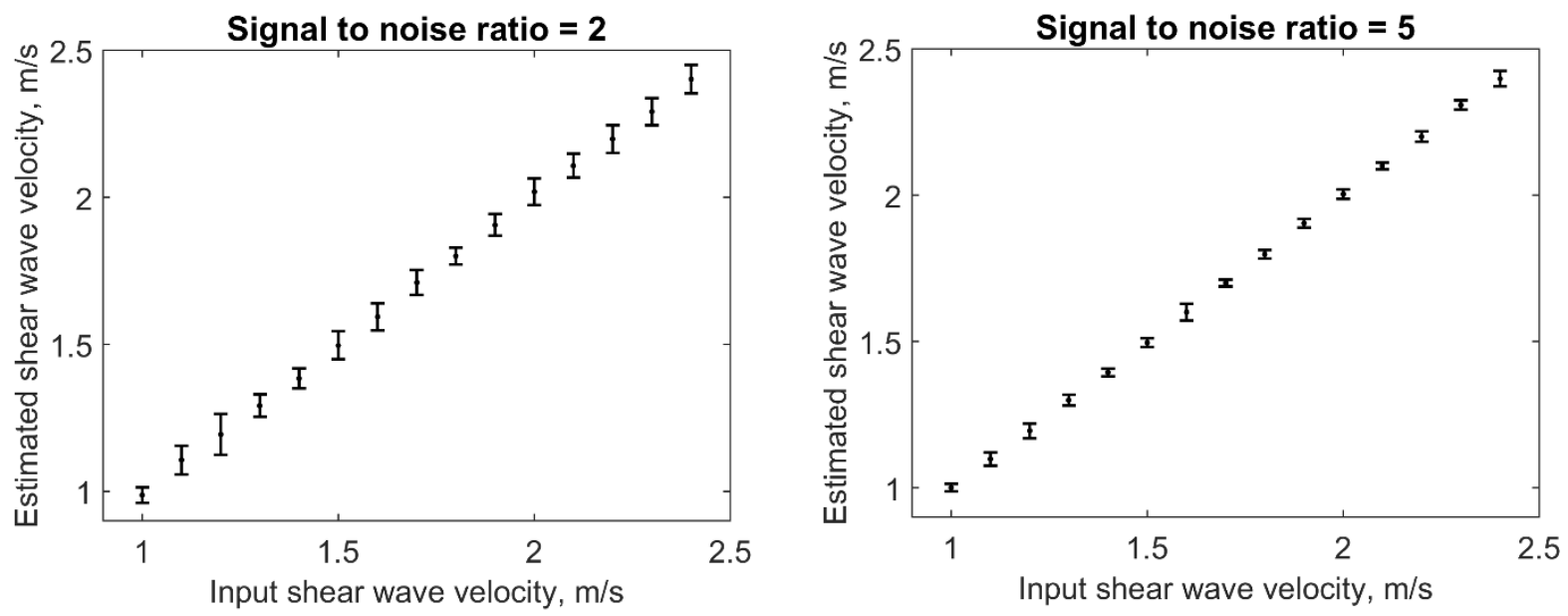

Figure 3: Shear wave velocity identified on numerical phantoms with varying elasticity, for two levels of signal to noise ratio. For each shear wave velocity value, 20 numerical phantoms were generated. Dots and errorbars denote the average and standard deviation across these 20 cases, respectively.

\section{Experimental results on Muscle}

\section{Experimental protocol \#1: Comparison between experimental and simulated MR-ARFI profile with} varying phase shifts

The delay between the onset of the MSG and the ARFI push was varied with incremental steps of $0.5 \mathrm{~ms}$. Experimental and simulated normalized MR-ARFI profiles are represented in Figure 4 for delay values of $\mathrm{T}_{\text {Delay }}=0 \mathrm{~ms}, 1.5 \mathrm{~ms}, 3 \mathrm{~ms}$ and $4.5 \mathrm{~ms}$. During the procedure, temperature increase measured by MR Thermometry did not exceed $3{ }^{\circ} \mathrm{C}$, resulting in a limited energy deposition. 

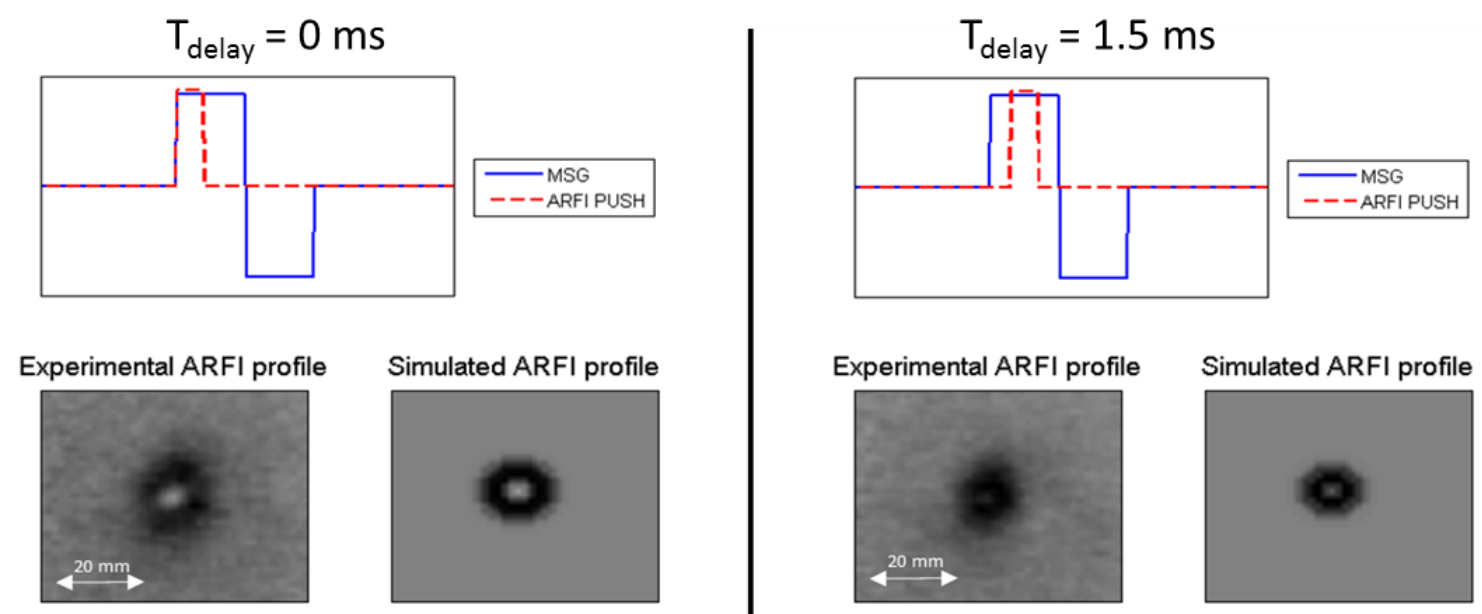

Simulated ARFI profile

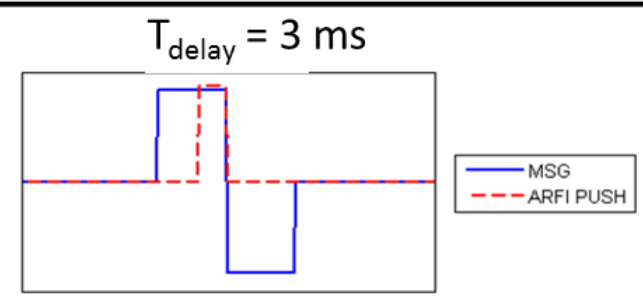

Experimental ARFI profile

Simulated ARFI profile
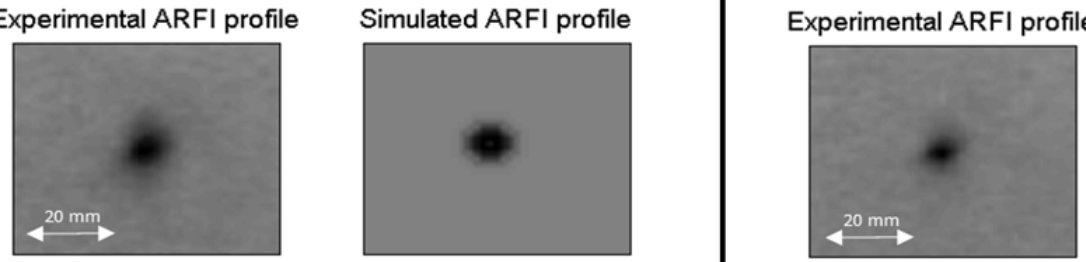

Simulated ARFI profile

Figure 4: Experimental and simulated normalized MR-ARFI profiles obtained for 4 different values of $\mathrm{T}_{\text {Delay }}$.

The same profiles can be represented in 1D after averaging along 4 radial directions: 

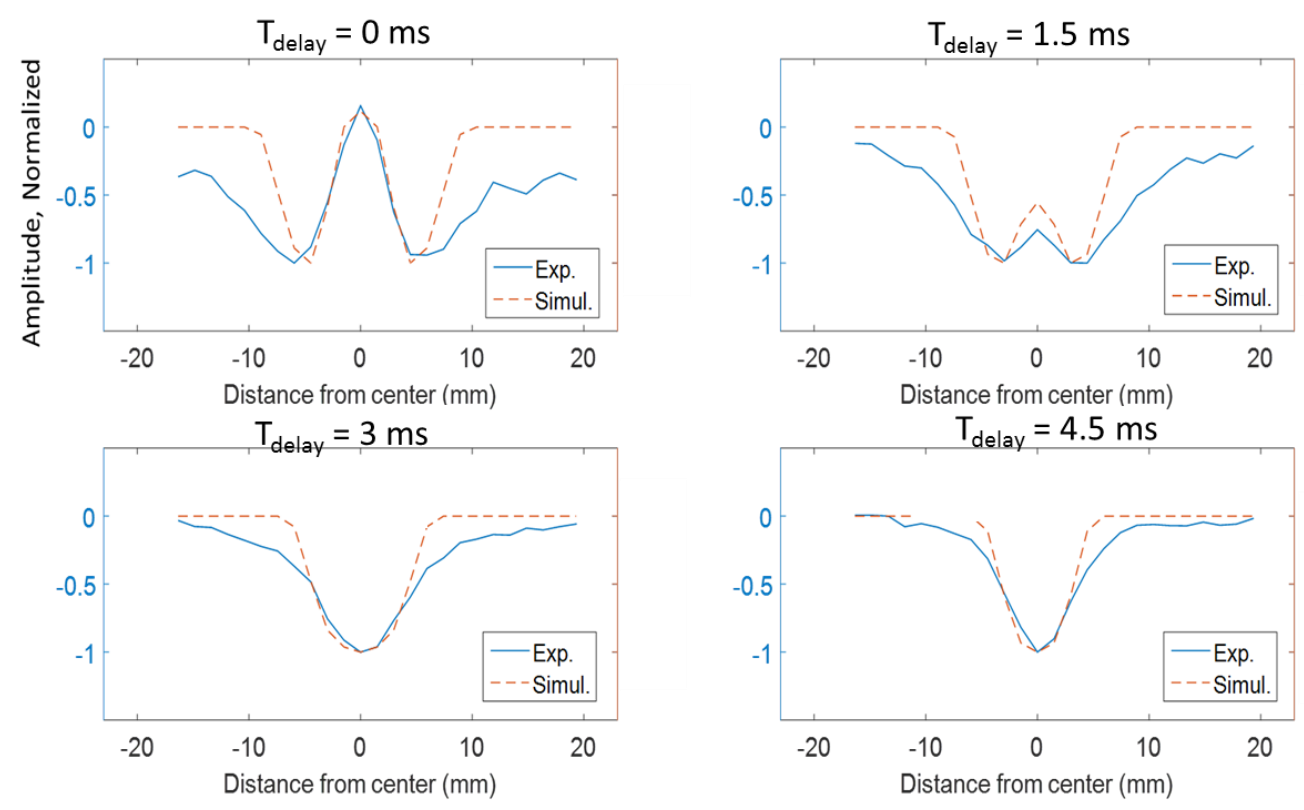

Figure 5: 1D profiles averaged along radial directions for the data presented in Figure 4, showing the experimental and simulated ARFI profiles for different values of $\mathrm{T}_{\text {Delay }}$.

These results illustrate the similarity between the experimental and the simulated MR-ARFI profiles for different $\mathrm{T}_{\text {Delay }}$, and the capability of the proposed ARFI simulator to properly compute the shape and the dimension of the ARFI pattern. Since the $\mathrm{T}_{\text {Delay }}$ of zero displays the maximal contrast, such a value was selected for the fitting process during HIFU ablation presented in the rest of the study.

\section{Experimental protocol \#2: Monitoring HIFU ablations}

The MR-ARFI "spot" was found to increase in size versus time in all of the six HIFU ablations, suggesting that the tissue stiffens as a result of the thermal lesion formation. An example of experimental and corresponding numerical MR-ARFI profiles are represented in Figure 6 at different ablation times. The full dynamic is available as a video in the supplementary material. Temperature elevation during the preablation step was negligible $\left(<1^{\circ} \mathrm{C}\right)$. 

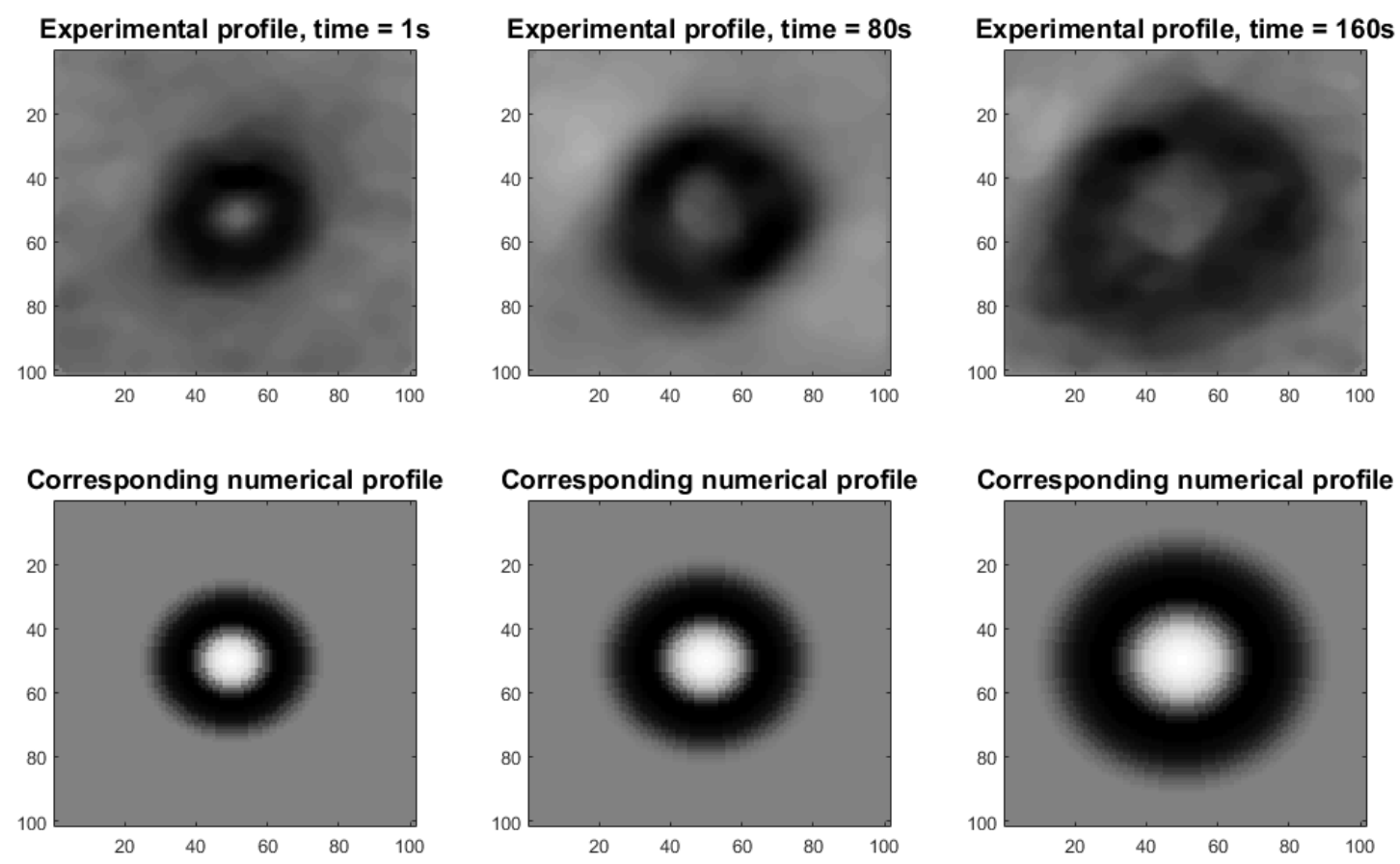

Figure 6: Examples of experimental MR-ARFI profiles at different ablation times for case \#1, and numerical profiles obtained by fitting the theoretical MR-ARFI model to experimental data. The color scale is the same $[-1 ; 1]$ and corresponds to normalized displacement fields.

Corresponding changes in the elastic modulus and in temperature are represented in Figure 7.
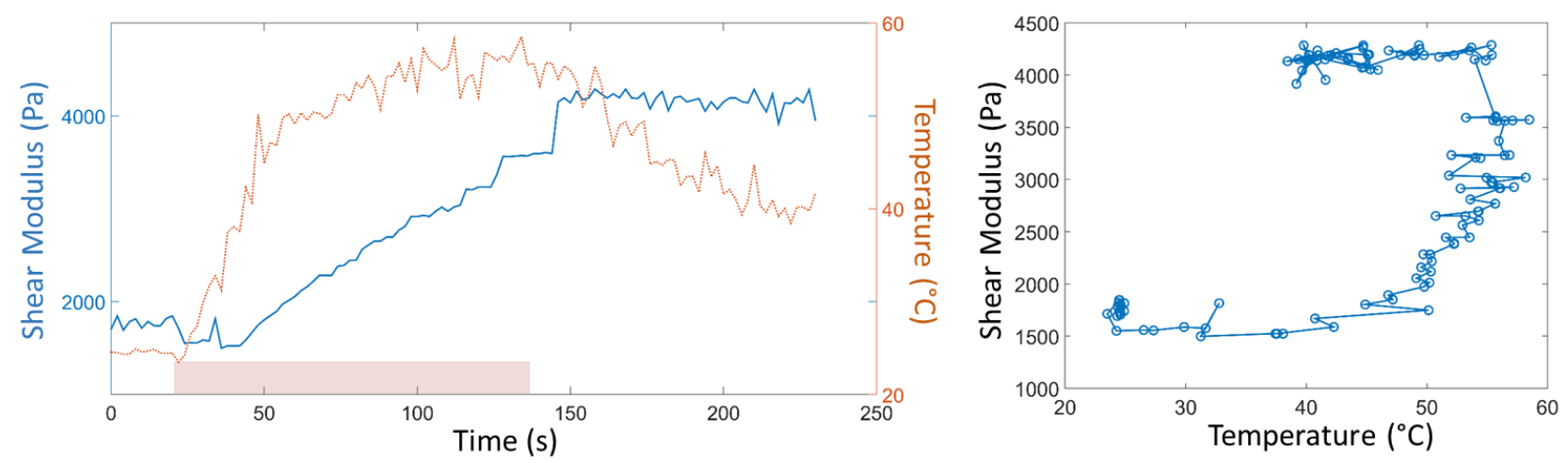

Figure 7: Example of the evolution of the shear modulus and of the temperature versus time (left); Shear modulus plotted against temperature (right). Timing of the HIFU sonication is represented by the red box 
Plotting changes in shear modulus against temperature allows observing the different phases of the experiment: First, temperature increases dramatically without any significant changes in stiffness. Stiffness starts increasing slightly towards the end of the ablation. Once ablation is over, temperature starts decreasing, whereas stiffness continues to increase until it reaches its final value.

\section{Discussion}

This study proposes a framework that allows computing quantitative MR-ARFI profiles depending on several experimental parameters, such as tissue elasticity, $\left(\mathrm{S}_{\mathrm{ARFI}}\right)$ pulse duration, synchronization between the ARFI push and the MSG $\left(\mathrm{T}_{\text {Delay }}\right)$, and properties of MSG.

The fitting process was first evaluated on numerical phantoms with different levels of added noise. Original elasticity was estimated with high accuracy, with low bias and low standard deviation for SNR down to 2. This numerical study allowed establishing the robustness and the reliability of the identification process.

Simulated MR-ARFI profiles were first compared to experimental ARFI pushes obtained with varying time delays between the ARFI push and MSG. Although the proposed method is effective for any value of $\mathrm{T}_{\text {Delay, }}$ the fitting process for tissue elasticity identification was found to be the most robust for $\mathrm{T}_{\text {Delay }}=0 \mathrm{~ms}$, i.e., when the ARFI push starts with the first lobe of the MSG. This may be explained by the fact that the corresponding profile is bipolar (positive and negative peaks, cf Figure 5), which constrains the fitting 
process and renders it more specific. Very good agreement between experimental and simulated MR-ARFI profiles allowed validating the proposed simulation framework. The MR-ARFI simulator was then used to fit experimental profiles obtained during HIFU ablations on porcine muscle tissue. This allowed identifying changes in tissue elasticity at every pair of MR images received. This study illustrates the value of tissue stiffness as a biomarker for tissue thermal damage. As opposed to temperature, it is directly linked to tissue damage, since tissue stiffness changes remain permanent even after HIFU ablation is turned off. Tissue stiffness measured with the proposed method appears as a complementary biomarker to thermal dose. The accuracy and the quantitative nature of the proposed method needs to be further investigated, for example through comparison with mechanical testing. However, it is already safe to claim at this stage that it does not suffer from some of the limitations of Thermal Dose, such as its high sensitivity to temperature uncertainty. Comparing these two biomarkers (Thermal Dose and ARFI-derived Elasticity) against histology would be highly interesting and is an obvious continuation of the present study.

Monitoring of thermal ablations requires flow of information in real-time in order to allow immediate action if needed. Although MRI acquisitions were performed fast enough for real-time control of the therapy $(\sim 1 \mathrm{~Hz})$, all elasticity estimations presented in this study were computed offline. However, translating the identification of the shear modulus in real time is feasible, using an online protocol similar to the one already proposed in interventional MRE (Corbin et al 2016). The computational time of the identification process varies currently between 1.5 and 3 seconds on a regular computer using the Matlab environment. This identification process should be optimized and shortened in order to allow for its use online in real time.

This study relies on several important assumptions that need to be reminded. First of all, this method assumes that tissue is locally isotropic, which may be questionable in several organs. The computed, simulated MR-ARFI profile does not depend on the direction of the wave propagation, and lead therefore to a circular MR-ARFI profile. Anisotropy would result in a non-circular profile. At this point, our method does not allow identifying tissue elasticity over several directions. However, the proposed MR-ARFI simulator could be extended to anisotropic media in order to identify tissue elasticity against direction of 
wave propagation $G(\theta)$, similarly to what has been proposed in quantitative, anisotropic MRE (Chatelin $e t$ al 2016). The second major assumption behind this study is the nature of the acoustic radiation force that was assumed punctual in our simulator. In reality, the force applies within a focal zone that depends on transducer geometry and properties. In the present study, the width of the focal zone at $-3 \mathrm{~dB}$ was $1.8 \mathrm{~mm}$ in diameter, in the same range of the voxel size of the MR acquisition sequence. Thus, the assumption made on punctual excitation appears reasonable. The higher the quality of focusing is, the closer we are to the assumption of a quasi-punctual force. This assumption may explain the slight differences between the experimental and simulated MR-ARFI profiles (see Figure 4 and 5))

In the present study, the monitoring was performed on a single slice orthogonal to the direction of ultrasound propagation, with $5 \mathrm{~mm}$ thickness and positioned at the ultrasound focus. Fast single shot EPI sampling allowed us to average acquisitions (five times) to produce phase images of better quality, while maintaining a refresh rate of $1 \mathrm{~Hz}$. Further validation studies aiming at comparing elasticity to thermal dose may require improving the spatial coverage in order to prevent missing out-of-plane heating. From this perspective, already existing methods such as simultaneous multi-slice acquisitions would allow acquiring several slices without changing the proposed acquisition strategy and sequence parameters (Barth et al 2016, Borman et al 2016).

In this study, displacement consecutive to the ARFI push was assumed to follow a $1^{\text {st }}$ order exponential curve with a relaxation time of $4 \mathrm{~ms}$. This is in the order of magnitude of what has already been measured in previous MR-ARFI studies (Dadakova, T. et al 2015). Measuring this relaxation time using the method proposed by (Dadakova, T. et al 2015) is expected to improve our elasticity estimates, by setting a parameter that is currently unknown. Typical tissue relaxation times are significantly higher under stress relaxation or creep experiments (Qiu et al 2008). However, in our case, the impulse is particularly short, leading to a particular bandwidth containing high frequencies. Typical relaxation times in the order of the millisecond are not uncommon at high frequency deformation, as attested by the complex modulus spectrum found in soft tissues (Papazoglou et al 2012, Vappou et al 2007). In any case, this is a particularly complex issue 
that is beyond the scope of this study. In the present study, the assumption made on the relaxation time is not expected to influence significantly the identification of tissue elasticity: it will mainly influence the amplitude of the displacement. Since data processing is performed on normalized data, errors on relaxation times are expected to have minimal impact of the results.

The experimental results presented in this study illustrate that elasticity is an interesting biomarker for monitoring HIFU ablations. The general evolution of elasticity is in agreement with previous studies that have investigated changes in tissue elasticity during thermal ablations (Arnal et al 2011, Brosses et al 2010, Corbin, N. et al 2015). Whereas tissue temperature was found to increase immediately after HIFU was turned on, elasticity changes seemed to occur after a few seconds. As expected, tissue temperature decreased immediately after HIFU was turned off, as opposed to tissue elasticity, which continued to increase until it reached its final value. This feature of tissue elasticity makes it an interesting biomarker for tissue integrity. The G vs T curve (Fig. 7) was similar to those reported in the literature (Brosses et al 2010, Arnal et al 2011). As in these studies, very slight softening was found to occur during the initial phase of heating, although this finding should be considered with caution and would require additional experiments to evaluate its consistency. Using tissue biomechanics for monitoring thermal ablations has already been proposed by several research teams. Compared to displacement-based methods (E. Konofagou et al 2012, Bour et al 2017), the proposed method offers the advantage of providing a quantitative parameter directly related to tissue elasticity, as opposed to displacement, which depends highly on many parameters that are not controlled, such as mechanical boundary conditions. The proposed method could be considered as a shear-wave-based elastography method, since it relies of the estimation of the shear wave velocity at the focal region. As opposed to MRE or shear wave-based ultrasonic methods such as shear wave ARFI or Supersonic Shear Wave Imaging (SSI), the proposed method is optimized for local estimation of elasticity specifically at the focal region. This entails significant advantages over the aforementioned methods for specific applications such as monitoring HIFU ablations. However, the proposed method does not allow for mapping tissue elastic properties beyond the focal region, as opposed to MRE. It is expected to perform 
better at a localized, point-size scale, without the possibility for mapping tissue properties. Beyond its applications for ablation monitoring, the proposed method could also be interesting for quantitative, highly localized biomechanical characterization of soft tissues.

\section{Conclusion}

A new, MR-ARFI simulator has been developed that allows computing normalized MR-ARFI profiles. This tool was used to identify tissue's elastic modulus at the focal region. This identification process was assessed in vitro on muscle tissue and was found to be highly reliable. Elasticity changes were followed during a HIFU ablation, and were compared to temperature changes measured by MR-Thermometry. Shear modulus was found to increase consistently and steadily a few seconds after the heating started, and such changes were found to be irreversible. Thanks to its ability to perform quantitative elasticity measurements in a highly localized manner around the focal spot, this method is particularly interesting for the monitoring of HIFU ablations.

\section{Acknowledgements}

This work was partly funded by the French state funds managed by the ANR (within the Investissements d'Avenir programme for the Labex CAMI and TRAIL); Grant number: ANR-11-LABX-0004 and ANR10-LABX-57, ANR-10-IAHU-04 (IHU Liryc) and by the WorkPackage\#3 (Interventional Imaging) of the France Life Imaging (FLI) network.

\section{References}

Arnal B, Pernot M and Tanter M 2011 Monitoring of thermal therapy based on shear modulus changes: II. Shear wave imaging of thermal lesions IEEE Trans. Ultrason. Ferroelectr. Freq. Control $\mathbf{5 8}$ 1603-11

Auboiroux V, Viallon M, Roland J, Hyacinthe J-N, Petrusca L, Morel D R, Goget T, Terraz S, Gross P, Becker C D and Salomir R 2012 ARFI-prepared MRgHIFU in liver: Simultaneous mapping of 
ARFI-displacement and temperature elevation, using a fast GRE-EPI sequence Magn. Reson. Med. $68932-46$

Barth M, Breuer F, Koopmans P J, Norris D G and Poser B A 2016 Simultaneous multislice (SMS) imaging techniques Magn. Reson. Med. 75 63-81

Bing K F, Rotemberg V M, Palmeri M L and Nightingale K R 2009 Concurrent ARFI imaging and HIFU ablation using a diagnostic transducer array and ultrasound system with custom beam sequences Ultrasonics Symposium (IUS), 2009 IEEE International Ultrasonics Symposium (IUS), 2009 IEEE International (IEEE) pp 65-8

Borman P T S, Bos C, Boorder T de, Raaymakers B W, Moonen C T W and Crijns S P M 2016 Towards real-time thermometry using simultaneous multislice MRI Phys. Med. Biol. 61 N461

Bour P, Marquet F, Ozenne V, Toupin S, Dumont E, Aubry J-F, Lepetit-Coiffe M and Quesson B 2017 Real-time monitoring of tissue displacement and temperature changes during MR-guided high intensity focused ultrasound Magn. Reson. Med. 78 1911-21

Brosses E S, Gennisson J-L, Pernot M, Fink M and Tanter M 2010 Temperature dependence of the shear modulus of soft tissues assessed by ultrasound Phys. Med. Biol. 551701

Chatelin S, Charpentier I, Corbin N, Meylheuc L and Vappou J 2016 An automatic differentiation-based gradient method for inversion of the shear wave equation in magnetic resonance elastography: specific application in fibrous soft tissues Phys. Med. Biol. 61 5000-19

Chen J, Woodrum D A, Glaser K J, Murphy M C, Gorny K and Ehman R 2013 Assessment of in vivo laser ablation using MR elastography with an inertial driver Magn. Reson. Med. n/a-n/a

Corbin, N., Vappou, J., Breton, E., Barbé, L., Renaud, P. and de Mathelin, M. 2015 Interventional Magnetic Resonance Elastography for MRI-guided percutaneous procedures ISMRM 23rd Annual Meeting \& Exhibition (Toronto, Canada)

Corbin N, Vappou J, Breton E, Boehler Q, Barbé L, Renaud P and de Mathelin M 2016 Interventional MR elastography for MRI-guided percutaneous procedures Magn. Reson. Med. 75 1110-8

Dadakova T, Krafft A J, Özen A C and Bock M 2017 Optimization of acoustic radiation force imaging: Influence of timing parameters on sensitivity Magn. Reson. Med. 10.1002/mrm.26734

Dadakova, T., Ozen, A.C., Krafft, A. J., Fuetterer, J., Hoogenboom, M., Jenne, J. W., Dumont, E., Damianou, C., Korvink, J.G. and Bock, M. 2015 MR-ARFI for the Quantification of Tissue Elastic Properties Proc. Intl. Soc. Mag. Reson. Med. ISMRM (Toronto, Canada)

E. Konofagou E, Maleke C and Vappou J 2012 Harmonic Motion Imaging (HMI) for Tumor Imaging and Treatment Monitoring Curr. Med. Imaging Rev. 8 16-26

Elias W J, Huss D, Voss T, Loomba J, Khaled M, Zadicario E, Frysinger R C, Sperling S A, Wylie S, Monteith S J, Druzgal J, Shah B B, Harrison M and Wintermark M 2013 A Pilot Study of Focused Ultrasound Thalamotomy for Essential Tremor N. Engl. J. Med. 369 640-8

Gianfelice D, Gupta C, Kucharczyk W, Bret P, Havill D and Clemons M 2008 Palliative Treatment of Painful Bone Metastases with MR Imaging-guided Focused Ultrasound Radiology 249 355-63 
Glaser K J, Manduca A and Ehman R L 2012 Review of MR elastography applications and recent developments J. Magn. Reson. Imaging 36 757-774

Goldstein R M, Zebker H A and Werner C L 1988 Satellite radar interferometry: Two-dimensional phase unwrapping Radio Sci. 23 713-20

Hansen M S and Sørensen T S 2013 Gadgetron: An open source framework for medical image reconstruction Magn. Reson. Med. 69 1768-76

Ishihara Y, Calderon A, Watanabe H, Okamoto K, Suzuki Y, Kuroda K and Suzuki Y 1995 A precise and fast temperature mapping using water proton chemical shift Magn. Reson. Med. 34 814-23

Larrat B, Pernot M, Aubry J-F, Dervishi E, Sinkus R, Seilhean D, Marie Y, Boch A-L, Fink M and Tanter M 2010 MR-guided transcranial brain HIFU in small animal models Phys. Med. Biol. 55365

Larrat B, Pernot M, Aubry J-F, Sinkus R, Tanter M and Fink M 2008 Radiation force localization of HIFU therapeutic beams coupled with Magnetic Resonance-Elastography treatment monitoring, In vivo application to the rat brain Proceedings - IEEE Ultrasonics Symposium pp 451-4

Le Y, Glaser K, Rouviere O, Ehman R and Felmlee J P 2006 Feasibility of simultaneous temperature and tissue stiffness detection by MRE Magn. Reson. Med. 55 700-5

Liberman B, Gianfelice D, Inbar Y, Beck A, Rabin T, Shabshin N, Chander G, Hengst S, Pfeffer R, Chechick A, Hanannel A, Dogadkin O and Catane R 2009 Pain Palliation in Patients with Bone Metastases Using MR-Guided Focused Ultrasound Surgery: A Multicenter Study Ann. Surg. Oncol. 16 140-6

Liu Y, Fite B Z, Mahakian L M, Johnson S M, Larrat B, Dumont E and Ferrara K W 2015 Concurrent Visualization of Acoustic Radiation Force Displacement and Shear Wave Propagation with 7T MRI PLoS ONE 10 e 0139667

Mariani A, Kwiecinski W, Pernot M, Balvay D, Tanter M, Clement O, Cuenod C A and Zinzindohoue F 2014 Real time shear waves elastography monitoring of thermal ablation: in vivo evaluation in pig livers J. Surg. Res. 188 37-43

McDannold N and Maier S E 2008 Magnetic resonance acoustic radiation force imaging Med. Phys. 35 $3748-58$

Mougenot C, Quesson B, de Senneville B D, de Oliveira P L, Sprinkhuizen S, Palussière J, Grenier N and Moonen C T W 2009 Three-dimensional spatial and temporal temperature control with MR thermometry-guided focused ultrasound (MRgHIFU) Magn. Reson. Med. 61 603-14

Muthupillai R, Rossman P J, Lomas D J, Greenleaf J F, Riederer S J and Ehman R L 1996 Magnetic resonance imaging of transverse acoustic strain waves Magn. Reson. Med. 36 266-274

Papazoglou S, Hirsch S, Braun J and Sack I 2012 Multifrequency inversion in magnetic resonance elastography Phys. Med. Biol. 57 2329-46

Payne A, de Bever J, Farrer A, Coats B, Parker D L and Christensen D A 2015 A simulation technique for 3D MR-guided acoustic radiation force imaging Med. Phys. 42 674-84 
Qiu Y, Sridhar M, Tsou J K, Lindfors K K and Insana M F 2008 Ultrasonic Viscoelasticity Imaging of Nonpalpable Breast Tumors: Preliminary Results Acad. Radiol. 15 1526-33

Quesson B, de Zwart J A and Moonen C T W 2000 Magnetic resonance temperature imaging for guidance of thermotherapy J. Magn. Reson. Imaging 12 525-533

Rieke V and Butts Pauly K 2008 MR thermometry J. Magn. Reson. Imaging 27 376-390

Roujol S, Ries M, Quesson B, Moonen C and Denis de Senneville B 2010 Real-time MR-thermometry and dosimetry for interventional guidance on abdominal organs Magn. Reson. Med. 63 1080-7

Sapareto S A and Dewey W C 1984 Thermal dose determination in cancer therapy Int. J. Radiat. Oncol. $10787-800$

Schmitz A C, Gianfelice D, Daniel B L, Mali W P T M and Bosch M A A J van den 2008 Image-guided focused ultrasound ablation of breast cancer: current status, challenges, and future directions Eur. Radiol. 18 1431-41

Souchon R, Salomir R, Beuf O, Milot L, Grenier D, Lyonnet D, Chapelon J and Rouvière O 2008 Transient MR elastography (t-MRE) using ultrasound radiation force: Theory, safety, and initial experiments in vitro Magn. Reson. Med. 60 871-81

Vappou J, Breton E, Choquet P, Goetz C, Willinger R and Constantinesco A 2007 Magnetic resonance elastography compared with rotational rheometry for in vitro brain tissue viscoelasticity measurement Magn. Reson. Mater. Phys. Biol. Med. 20 273-8

Vappou J, Hou G Y, Marquet F, Shahmirzadi D, Grondin J and Konofagou E E 2015 Non-contact, ultrasound-based indentation method for measuring elastic properties of biological tissues using Harmonic Motion Imaging (HMI) Phys. Med. Biol. 602853

Weidensteiner C, Kerioui N, Quesson B, de Senneville B D, Trillaud H and Moonen C T W 2004 Stability of real-time MR temperature mapping in healthy and diseased human liver J. Magn. Reson. Imaging 19 438-46

Wu T, Felmlee J P, Greenleaf J F, Riederer S J and Ehman R L 2001 Assessment of thermal tissue ablation with MR elastography Magn. Reson. Med. 45 80-87

Zaaroor M, Sinai A, Goldsher D, Eran A, Nassar M and Schlesinger I 2017 Magnetic resonance-guided focused ultrasound thalamotomy for tremor: a report of 30 Parkinson's disease and essential tremor cases J. Neurosurg. 1-9 